\title{
Deciduous tree reconstruction algorithm based on cylinder fitting from mobile terrestrial laser scanned point clouds
}

\author{
Valeriano Méndez , Joan Ramon Rosell-Polo , Ricardo Sanz , \\ Alexandre Escolà , Heliodoro Catalán
}

\begin{abstract}
Vector reconstruction of objects from an unstructured point cloud obtained with a LiDARbased system (light detection and ranging) is one of the most promising methods to build three dimensional models of orchards. The cylinder fitting method for woody structure reconstruction of leafless trees from point clouds obtained with a mobile terrestrial laser scanner (MTLS) has been analysed. The advantage of this method is that it performs reconstruction in a single step. The most time consuming part of the algorithm is generation of the cylinder direction, which must be recalculated at the inclusion of each point in the cylinder. The tree skeleton is obtained at the same time as the cluster of cylinders is formed. The method does not guarantee a unique convergence and the reconstruction parameter values must be carefully chosen. A balanced processing of clusters has also been defined which has proven to be very efficient in terms of processing time by following the hierarchy of branches, predecessors and successors. The algorithm was applied to simulated MTLS of virtual orchard models and to MTLS data of real orchards. The constraints applied in the method have been reviewed to ensure better convergence and simpler use of parameters. The results obtained show a correct reconstruction of the woody structure of the trees and the algorithm runs in linear logarithmic time.
\end{abstract}

\section{Introduction}

Geometric reconstruction can be used to obtain a detailed structural analysis of trees. The aim is to derive vegetative parameters such as leaf area, canopy volume or woody volume from massive data point clouds. Direct use of raster information, e.g. a photograph, can be used to obtain any of these parameters (Phattaralerphong \& Sinoquet, 2007). Reconstruction of tree geometry supports the implementation 


\begin{tabular}{|c|c|c|c|}
\hline \multicolumn{2}{|c|}{ Nomenclature } & $n_{\min }$ & Minimum number of points used to determine the \\
\hline \multicolumn{2}{|c|}{ Variable Description } & & significant parent or predecessor branch \\
\hline \multirow{2}{*}{ A } & Covariance matrix & $n_{p}$ & Number of points of the considered parent or \\
\hline & $\frac{\text { Polar angle used in the iterative method to obtain }}{\vec{d}}$ & $n_{s}$ & $\begin{array}{l}\text { predecessor branch } \\
\text { Number of points that freely seed a cylinder when }\end{array}$ \\
\hline \multirow{2}{*}{$\begin{array}{l}B \\
B^{*}\end{array}$} & A branch object & & the bulding of a new branch starts \\
\hline & $\begin{array}{l}\text { Temporal branch built when a new point is } \\
\text { included in the process }\end{array}$ & 0 & $\begin{array}{l}\text { An upper limit of growth of the algorithm } \\
\text { response time }\end{array}$ \\
\hline$B N$ & A new branch built by the branching process & ord & Branching order according to the terminology \\
\hline \multirow{2}{*}{$\stackrel{c}{\vec{d}}$} & Centroid of a branch & & proposed by De Reffye, Edelin, Jaeger, and Puech \\
\hline & Cylinder direction of a branch & & Order of the checked narent or predecessor \\
\hline $\overrightarrow{d^{*}}$ & $\begin{array}{l}\text { Cylinder direction of a branch estimated by a } \\
\text { numerical method }\end{array}$ & ord $_{C}$ & branch used to determine the significant parent or \\
\hline$\Delta \alpha$ & Polar angle resolution used in iterative method to & $\operatorname{ord}_{\min 1}$ & predecessor branch \\
\hline \multirow[t]{2}{*}{$\Delta \varphi$} & Azimuthal angle resolution used in iterative & $\operatorname{ord}_{\min 2}$ & $\begin{array}{l}\text { Rank of order used to determine the significant } \\
\text { parent or predecessor branch }\end{array}$ \\
\hline & metnoa to odtain a & $P$ & An individual point of the point cloud \\
\hline & MTLS longitudinal resolution (distance between & $P_{1}$ & $\begin{array}{l}\text { Initial point of the cylinder axis that models a } \\
\text { branch }\end{array}$ \\
\hline$\varphi$ & $\begin{array}{l}\text { vertical scans) } \\
\text { Azimuthal angle used in iterative method to } \\
\text { obtain } \vec{d}\end{array}$ & $P_{2}$ & $\begin{array}{l}\text { Final point of the cylinder axis that models a } \\
\text { branch }\end{array}$ \\
\hline \multirow{4}{*}{$\begin{array}{l}\text { GNSS } \\
\text { HMT } \\
k_{r}\end{array}$} & Global navigation satellite system & $P_{d}$ & Projection of $\mathrm{P}$ over the cylinder axis in a branch \\
\hline & Hidden Markov tree & $P_{r}$ & Initial point, placed at the base of the trunk, taken \\
\hline & Factor of radius $r$ to determine whether $P$ is & $\theta$ & $\begin{array}{l}\text { as origin of the tree model reconstruction. } \\
\text { Angular position of laser beam }\end{array}$ \\
\hline & $\begin{array}{l}\text { aligned in current branch } B \text { or allows a new branch } \\
B N\end{array}$ & $r$ & Radius of the cylinder that models a branch \\
\hline \multirow{7}{*}{$\begin{array}{l}1 \\
\text { MTLS } \\
M \\
N \\
n \\
n_{b}\end{array}$} & Distance from the laser sensor to a tree object & SCA & Space colonisation algorithm \\
\hline & Mobile terrestrial laser scanning & TIN & Triangulated irregular network \\
\hline & Directions to the centroid matrix & $t_{2}$ & Value of parameter $t$ for $P_{2}$ in a vector straight \\
\hline & Number of points in the point cloud & & equation defined by $P_{1}$ and $d$ \\
\hline & Number of points in a branch or cylinder & $t_{d}$ & Value of parameter $t$ for $P_{d}$ in a vector equation of a \\
\hline & Number of branches & $y$ & MTLS longitudinal position \\
\hline & & $\mathrm{z}_{0}$ & Height of the laser sensor \\
\hline
\end{tabular}

of virtual tree models, such as use of the statistical framework of the hidden Markov tree (HMT) model introduced by Crouse, Nowak, and Baraniuk (1998) and used for constructing realistic apple trees by Durand, Guédon, Caraglio, and Costes (2005) and Costes et al. (2008).

In parallel with the use of massive data from photogrammetry or aerial scanning for the detection of trees and estimation of their general parameters, two main approaches are used to study their geometry at individual tree level. The first is based on digital photographs (Mizoue \& Masutani, 2003; Phattaralerphong \& Sinoquet, 2005, 2007; Shlyakhter, Rozenoer, Dorsey, \& Teller, 2001; Tan, Fang, Xiao, Zhao, \& Quan, 2008): graphic data are processed to determine the existence of vegetation and sensor parameters (camera height and its horizontal distance to the tree) allow a projection to be obtained on a voxel space, with which the tree-top and leaf area can be estimated (Phattaralerphong \& Sinoquet, 2007). The use of a reduced voxel size to improve accuracy dramatically increases the processing time.

The second approach uses mobile terrestrial laser scanning (MTLS) to obtain a dense point cloud from which a detailed geometrical description can be extracted (Rosell et al. 2009; Sanz-Cortiella et al. 2011). Simonse, Aschoff, Spiecker, and Thies (2003) detected woody geometry from MTLS data using the Hough transform and Gorte and Winterhalder (2004) as well as Pfeifer, Gorte, and Winterhalder (2004) created a topology skeleton from a voxel space. The use of TIN (triangulated irregular network) to obtain geometric information about woody tree structure is limited by stem capillarity (Fig. 1) and usually supports extraction of neighbourhood graphs (adjacency relations between all the points). Pfeifer et al. (2004) obtained a model of major branches and stems with cylinder fitting. Other methods, which combine scanning data with texture information from high resolution photographs, have been proposed by Reulke and Haala (2005). Iterative closest point (ICP) algorithms have also been used to fit the guide lines obtained in different scans (Besl \& McKay, 1992; Henning \& Radtke, 2006). The algorithm iteratively revises the geometric transformation needed to minimise the distance between the points of the different raw scans.

It is easy to determine whether a point of the MTLS point cloud belongs to the trunk and main branches. However in the lowest branches, particularly the stems, it becomes more 


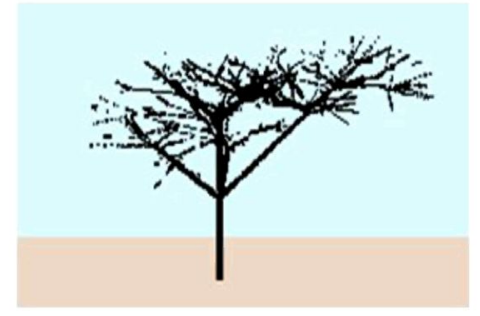

a

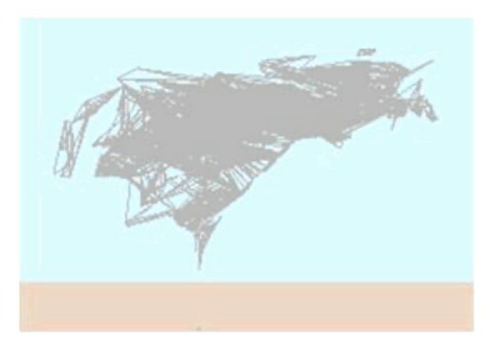

b

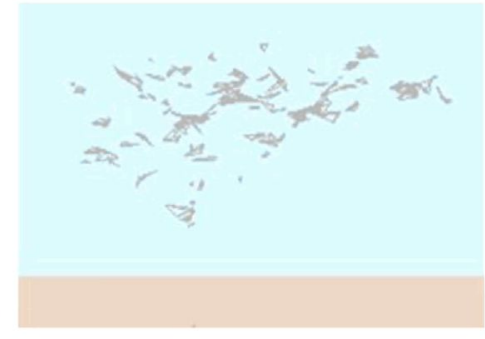

C

Fig. 1 - MTLS unstructured point cloud simulated with SIMLIDAR (a), where a triangulated irregular network (TIN) has been calculated. The broad capillarity prevents reconstruction through filtering of initial tetrahedrons (b) by size (c) could be used to characterize the stems structure.

difficult to determine whether a point of the cloud belongs to one stem or another. Neighbourhood graphs, geodesic graphs and several clustering algorithms can be used to obtain the skeleton of the tree and the radius of each branch. The search of points to build neighbourhood graphs is based on $\mathrm{kd}$-tree, a $\mathrm{k}$-dimensional binary tree generated by hyperplane splitting that divides the space in two half-spaces. Verroust and Lazarus (2000) generated the skeleton of a tree from a set of neighbour graphs, geodesic graphs (selecting an initial point at the base of the trunk, $P_{r}$, and the shortest path from each point to $P_{r}$ ) and k-levels (defined by Lloyd (1982) which divide the graph into clusters of close points). From a kd-tree, Yan et al. (2009) applied the Lloyd iteration (1982) to obtain a segmentation of the cloud in clusters based on cylinders. Delagrange and Rochon (2011) used the model of Verroust and Lazarus (2000) to obtain the skeleton and select centroids within it. They then applied a clustering process to connect each point to their respective branch. The vector reconstruction method proposed by Verroust and Lazarus (2000) or Delagrange and Rochon (2011) requires executing the process in stages: neighbourhood graph, geodesic graph, skeleton extraction, skeleton population with adjacent points clusters and, finally, fitting each cluster with a surface. Preuksakarn et al. (2010) use a space colonisation algorithm (SCA) as a function of clustering. De Aguiar, Stoll, et al., 2008 and De Aguiar, Theobalt, et al., 2008, use clustering processes to capture shapes from video data.

In this work, the approach proposed by Pfeifer et al. (2004) is used as a direct algorithm for woody structure reconstruction. One of the objectives was to minimize the number of parameters that control the operation of the algorithm. The existence of a large number of empirical parameters controlling the process can distort the method and make it more difficult to attain the desired unique solution. The developed algorithm was applied to point clouds obtained from MTLS measurements of real orchards and point clouds obtained from simulated MTLS measurements of virtual orchards built with SIMLIDAR software (Méndez et al., 2012; Méndez, Catalán, Rosell, Arnó, \& Sanz, 2013), respectively. Models of woody trees with a high degree of branching, applicable to deciduous leaf species, were used. Simulations with varying degrees of scanning density were also tested.

The information provided by this algorithm could be useful for the modelling of orchards and their evolution from both a scientific and commercial perspective. Using MTLS of trees and subsequently obtaining and quantifying the woody structure with the proposed algorithm at the beginning of the season can help growers and/or advisors to:

- Improve the determination of seasonal foliage evolution by subtracting the woody model from the MTLS point clouds obtained during the season. Knowing the leaf area is very useful in terms of plant protection products dosage and canopy management in general.

- Decide on pruning intensity by comparing the woody model obtained at the end of the season with the one obtained at the end of the previous season. Additionally, scanning the trees before and after pruning can help growers see the potential effect of pruning intensity on the next season's production.

- Check whether tree growth is correct in terms of its evolution over the seasons and in terms of its training system.

- Estimate the total volume of the ligneous fraction of the tree orchard and its evolution over the years, constituting a novel approach for other agricultural research purposes.

\section{Materials and methods}

\subsection{Data}

The proposed algorithm was applied to real MTLS data from a pear orchard and to simulated MTLS data of an apple orchard and a vineyard virtually obtained with SIMLIDAR software (Fig. 2a and b).

The real MTLS operation was performed on a cv. Blanquilla pear orchard (Pyrus communis L. 'Blanquilla') after leaf-fall (see Fig. 2c). A Fiatagri 80-76 DT tractor model was used at a forward speed of $1 \mathrm{~km} \mathrm{~h}^{-1}$. The sensor was placed at a height of $2.10 \mathrm{~m}$, angular resolution $(\Delta \theta)$ was set to $1^{\circ}$ and longitudinal resolution was $15 \mathrm{~mm}$ (distance between vertical scans).

The simulated MTLS operation was applied to a virtual apple orchard obtained with SIMLIDAR software (Méndez et al., 2013), based on a HMT modelling process (Durand et al. 2005) and to a virtual vineyard based on a SIMLIDAR generated growth pattern. A simulated monolateral MTLS using SIMLIDAR (Méndez et al. 2012, 2013) was applied to both 


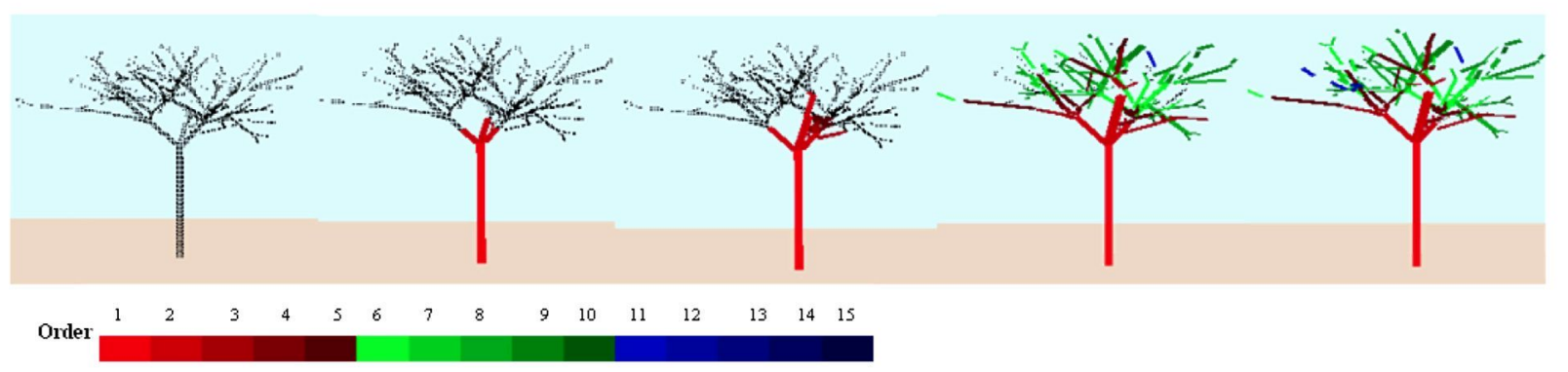

(a)

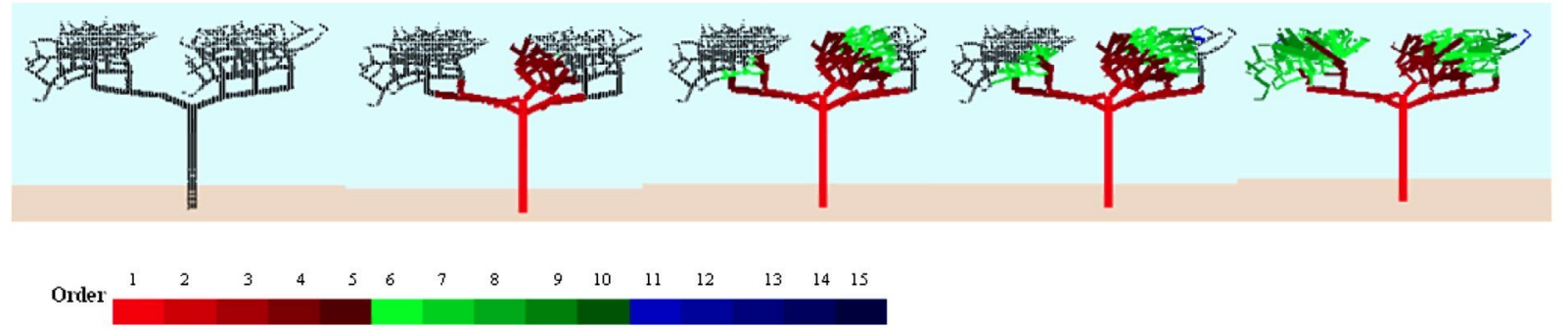

(b)

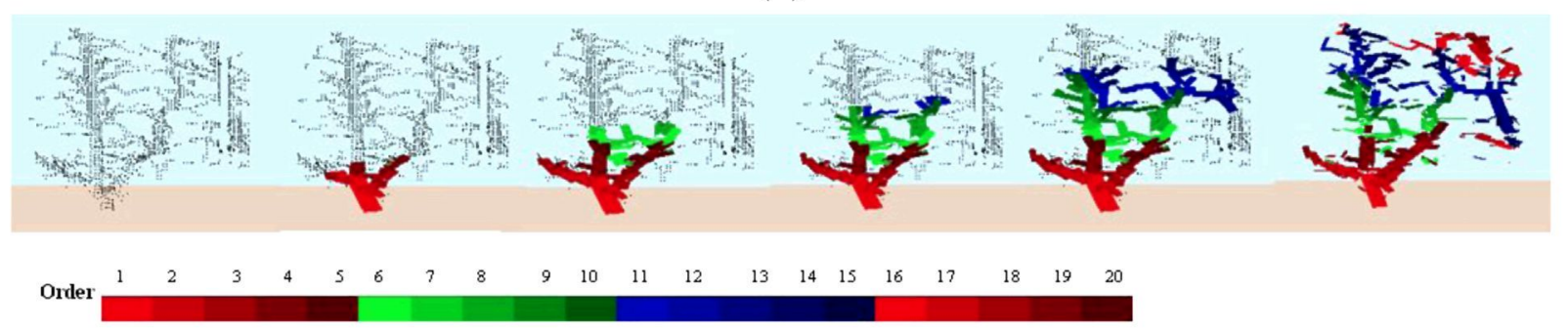

(c)

Fig. 2 - Reconstructions of a virtual apple-tree (a) and vineyard (b) from their simulated MTLS. Reconstruction of a real peartree (c) from their MTLS. The order number is represented as cycles of red, green and blue colours.

virtualisations with an angular resolution of $0.5^{\circ}$ and a longitudinal resolution of $10 \mathrm{~mm}$.

\subsection{Algorithm}

The algorithm was developed in Microsoft ${ }^{[}$Visual $\mathrm{C}++$ and run on a PC (HP ${ }^{\mathbb{B}}$ Compaq dc 7700p, Intel(R) Core(TM)2 CPU $6600,2.40 \mathrm{GHz}, 3.49 \mathrm{~GB}$ RAM with a Windows ${ }^{\mathbb{E}} \mathrm{XP}$ Professional operating system).

MTLS provides distances (l) from the sensor to each tree object, at a given vehicle longitudinal advance position $(y)$ and at an angular value of the sensor's emitted beam direction $(\theta)$. For each scan, the acquisition system stores the triplet $\left(y_{i} \theta_{i} l_{i}\right)$ with $i=1 \cdots N$ (where $N$ is the total number of measurements). From a set of $\left(y_{i} \theta_{i} l_{i}\right)$ and knowing the longitudinal advance increment $(\Delta y)$, the angular resolution $(\Delta \theta)$ and the height of the sensor $\left(z_{0}\right)$, it is possible to obtain the 3D coordinates $\left(x_{i} y_{i} z_{i}\right)$ of each intercepted point of the tree. By using a global navigation satellite system (GNSS) to determine the sensor position for each scan, it is possible to obtain the absolute coordinates for each point in the point cloud.
Although a lateral MTLS intercepts all the geometrical data of an orchard, its operation is optimum in a sparsely populated structure, as is the case with agricultural deciduous species. When using a bilateral or multilateral scanner, the problem of measurement errors increases significantly, with a dead-reckoning system for the accumulated errors not being possible (Guivant, Nebot, \& Durrant-Whyte, 2002; Nebot \& Durrant-Whyte, 1999; Neira, Tardos, \& Castellanos, 2003). In this case it is essential to use reference points, or guidance systems based on a SLAM algorithm (simultaneous localisation and mapping, Auat Cheein \& Guivant, 2014; Iagnemma, Kang, Shibly, \& Dubowsky, 2004) to statistically estimate the dragged errors.

The work starts with an unstructured point cloud, with all the inner points consistent after a debugging process. The "cylinder following" method proposed by Pfeifer et al. (2004) aims to build the skeleton, simultaneously populating the cylinders with adjacent points, without using a prior neighbourhood or geodesic graph. It is based on constructing a cylinder that fits the trunk of the tree and a cylinder vector structure, which extends upwards and outwards, that is fitted through all the points of the cloud to obtain a populated skeleton that is the woody structure. 


\subsection{Setting cylinder direction}

Setting the direction of the cylinder requires determining the cylinder which best fits a set of points. Given a set of points $S=\left\{P_{i}=\left(x_{i} y_{i} z_{i}\right)\right\}$ with $i=1, \cdots, n$, with $n$ being the number of points of the cylinder, the cylinder trunk that best fits $S$ will have an axis that goes through the centroid $c$ of $S$, with $\mathrm{c}=\left(\begin{array}{lll}\bar{x} & \bar{y} & \bar{z}\end{array}\right)=\frac{1}{n} \sum_{i=1}^{n}\left(\begin{array}{lll}x_{i} & y_{i} & z_{i}\end{array}\right)$. If the cylinder axial direction $\vec{d}=\left(\begin{array}{lll}d_{x} & d_{y} & d_{z}\end{array}\right)$ is the direction that minimises the maximum of orthogonal distances $\left(P_{i}, \vec{d}\right)$, it is possible to obtain $\vec{d}$ with an iterative method (Rabbani \& Heuvel, 2005), taking directions with angles $(\alpha \varphi)$ with $0 \leq \alpha \leq \pi, 0 \leq \varphi \leq 2 \pi$ and successively changing $\Delta \alpha$ and $\Delta \varphi$ until finding where the orthogonal distances are minimum.

It is also possible to obtain $\vec{d}$ as a non-linear least-squares estimate (Lukács, Martin, \& Marshall, 1998; Marshall, Lukács, \& Martin, 2001) as an eigenvector of a covariance matrix $A=M^{t} M$, where the ith row of $M$ is $p_{i}-c$, that is:

$M=\left(\begin{array}{ccc}x_{1}-\bar{x} & y_{1}-\bar{y} & z_{1}-\bar{z} \\ x_{2}-\bar{x} & y_{2}-\bar{y} & z_{2}-\bar{z} \\ \vdots & \vdots & \vdots \\ x_{N}-\bar{x} & y_{N}-\bar{y} & z_{N}-\bar{z}\end{array}\right)$

The matrix $A$ has a maximum of three eigenvectors that fit three cylindrical adjustments to the point cloud, taking the best direction as the one related to the lowest eigenvalue. The eigenvalues and eigenvectors can be calculated using the Rayleigh-Ritz ratio.

\subsection{Branching criterion}

The algorithm, shown in Table 1, starts by selecting an initial point at the base of the trunk $\left(P_{r}\right)$, with the condition that $P_{r}$ has a minimum value in $z$. The method continues in Table 2 to search for points close to $P_{r}$ setting a cylinder that fits the trunk, usually with the direction $\vec{d} \approx\left(\begin{array}{lll}0 & 0 & 1\end{array}\right)$. Optionally, the points search can be supported in a kd-tree to improve processing time. Those points, close to the initial cylinder and aligned with their current direction, can be considered as a continuation of the trunk, otherwise they will be considered the origin of a new branch. The setting of the direction $\vec{d}$ in the starting stage of a new branch is the main weakness of the algorithm. The direction of the trunk, once $P_{r}$ has been selected, does not emerge immediately from the first clustering of points close to $P_{r}$. It is necessary to seed the cylinder with a number of close points $\left(n_{s}\right)$, without checking the alignment ratio of each one with respect to the parameters of the cylinder $\left(\vec{d}, P_{1}, P_{2}, r\right)$. The parameter $n_{s}$ is applicable to the initial trunk and to all new branches to be reconstructed in the model. The parameter $n_{S}$ must be selected considering the scanning density used to obtain the point cloud and the branching order following the biological terminology of De Reffye et al. (1988). The density of points in the cloud depends on the values of $\Delta y$ and $\Delta \theta$ adopted in the MTLS operation; the greater the density, the greater $n_{s}$. The value of $n_{s}$ decreases as branch order increases in the model, which implies a decrease in the radius and the density of scanned points.

In a ligneous structure, the radii of successor branches are smaller than that of their parent. This property is used as a constraint in the model. This restriction has the advantage of reducing the need to find a value of $n_{s}$ only for the formation of the main trunk, but the behaviour is correct only in the major branches, where the order is low. For higher orders, reconstruction becomes an unrealistic capillary-like structure as all dependent cylinders are forced to have a smaller radius. As an intermediate alternative, in Table 2, a restriction has been used so that the branches have a radius smaller than a predecessor branch which can be considered significant. A

Table 1 - Function with the main process of reconstruction.

\begin{aligned} \hline Function & MainProcess \\ \hline Input & Void \\ Output & Void \\ 1: & CreateKTree() \\ 2: & Branch $\leftarrow$ GetFootTree 0 \\ 3: & List_Branches.Insert(Branch) \\ 4: & Iter From I $=1$ To length(List_Branches) \\ 5: & Branch $\leftarrow$ List_Branches[I] \\ 6: & If Branch.Status = 0 Then \\ 7: & Status $\leftarrow$ FindTheClosestPoint(Branch, ClosestPoint) \\ 8: & If Status = 0 Then \\ 9: & Branch.Status $\leftarrow 1 \\$ 10: & Else If Status $=1$ Then // No Aligned, new branch \\ 11: & List_Branches.Insert(new CBranch(ClosestPoint)) \\ 12: & Else // Aligned, insert point in current branch \\ 13: & Branch.AddPoint(ClosestPoint) \\ 14: & End(If) \\ 15: & End(If) \\ 16: & End(I) \\ 17: & AlignedChildrenBranches() \\ 18: & While(ConnectAlignedBranches) \\ 19: & While(Clustering) \\ 20: & Return \end{aligned}


branch is considered significant if it meets one of the following two conditions:

$\operatorname{ord}_{C}<\operatorname{ord}_{\min 1}$

$\operatorname{ord}_{C}<\operatorname{ord} d_{\min 2} \cup n_{\mathrm{P}}>n_{\min }$

where $\operatorname{ord}_{C}$ is the order of the verified parent branch, ord $d_{\min 1}$ and ord $_{\min 2}$ are parameters with values of the order of the parent branch, $n_{P}$ is the number of points of one of the predecessor branches of the branch under construction and $n_{\text {min }}$ is the minimum number of points that the branch should have to consider it significant. The data model of the branch class used (CBranch) has the properties shown in Fig. 3.

Each branch, except the trunk, has a pointer to the predecessor or parent branch and from zero to $\mathrm{N}$ successor

Table 2 - Function that searches the nearest point to a branch (top) and the auxiliary function that gets the significant parent of a current branch (bottom).
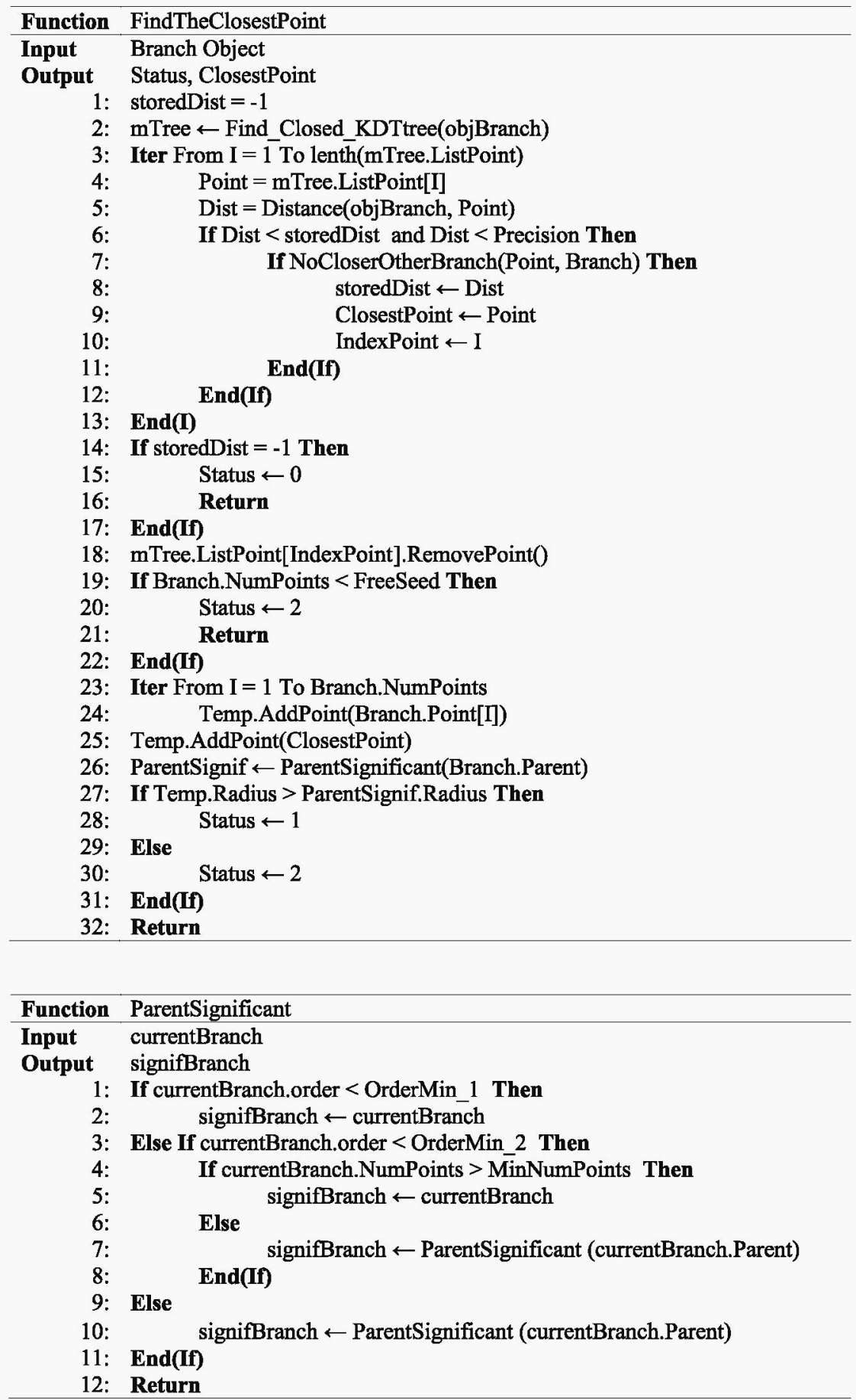


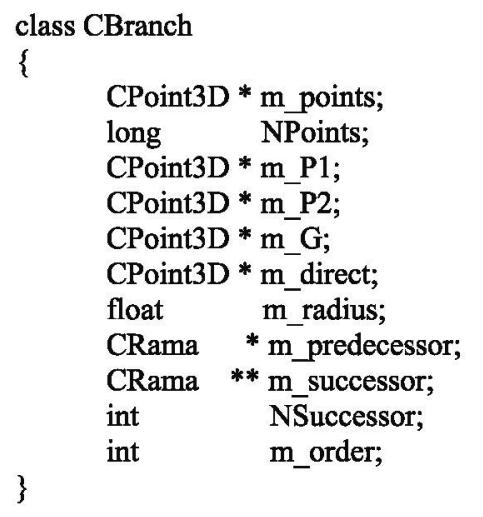

Fig. 3 - Data model of CBranch class.

branches. In the data structure, a pointer to the predecessor is provided, the value of which should be null for the main trunk which is the branch of order 1 . The successor branches, if any, are stored in an array of pointers. A significant branch is selected by moving back recursively in the predecessor hierarchy of a given branch, through the pointers of parents of following branches, searching for the predecessor that fulfils the minimum order $\left(o r d_{C}<\right.$ ord $d_{\text {min2 }}$ ) and a minimum number of points $\left(n_{\mathrm{p}}>n_{\min }\right)$. If this condition is not met in the hierarchy of predecessors, then the first branch that meets the condition ord $d_{C}<$ ord $d_{\min 1}$, with ord $d_{\min 1}<$ ord $d_{\min 2}$, is considered significant.

Determining if a point $P$ is aligned with the current branch and may be incorporated to a branch $B$ or whether it is necessary to start the building of a new branch $(B N)$ is a process that depends on the characteristics of the cylinder $B$ $\left(\vec{d}, P_{1}, P_{2}, r\right)$ and on the characteristics of $B^{*}$, with $B^{*}=B \cup P$. The cylinder generated by $B^{*}$ is characterised by $\vec{d}^{*}, P_{1}^{*}, P_{2}^{*}, r^{*}$. If $r^{*}>k_{r}{ }^{*} r$ with $k_{r}>1$, then it is considered that the point does not align and a new branch BN is started. The value of $k_{r}$ depends on the position of the point $P$ when it is projected on the branch. If $P_{d}$ is the projection on the straight line defined by $P_{1}$ and $\vec{d}$, then it will be true that $P_{d}=P_{1}+t_{d} * \vec{d}$. Furthermore, as $P_{2}$ is selected so that $P_{2}=P_{1}+t_{2} * \vec{d}$, where $t_{2}>0$, it results that $P_{1}<P_{2}$. Therefore, depending on the position of $P_{d}$ (or the value of $t_{d}$ ), different values of $k_{r}$ may be taken.

\subsection{Clustering}

The algorithm can make the mistake of considering that $P$ generates a new branch $B N$ when it is actually a mere bulge of $B$. In addition, from this mistaken new branch $B N$, a thread is reconstructed that actually belongs to the predecessor branch. The multithreading problem is solved with two alternative clustering processes. The first process, shown in Table 3 , detects successor branches of one predecessor with a similar direction $\vec{d}$ between them and merges them all. The second process, shown in Table 4, detects a predecessor branch and one successor branch that must also be a continuation of each other and forms a single cylinder.

Finally a balanced clustering process, also following the hierarchy between each branch and its successors, is adopted as shown in Table 5. It is considered that the tree structure must be optimal, in other words that its main geometric parameters must be minimum. Then the points between a

Table 3 - Function that joints a set of children branches that get a one aligned branch.

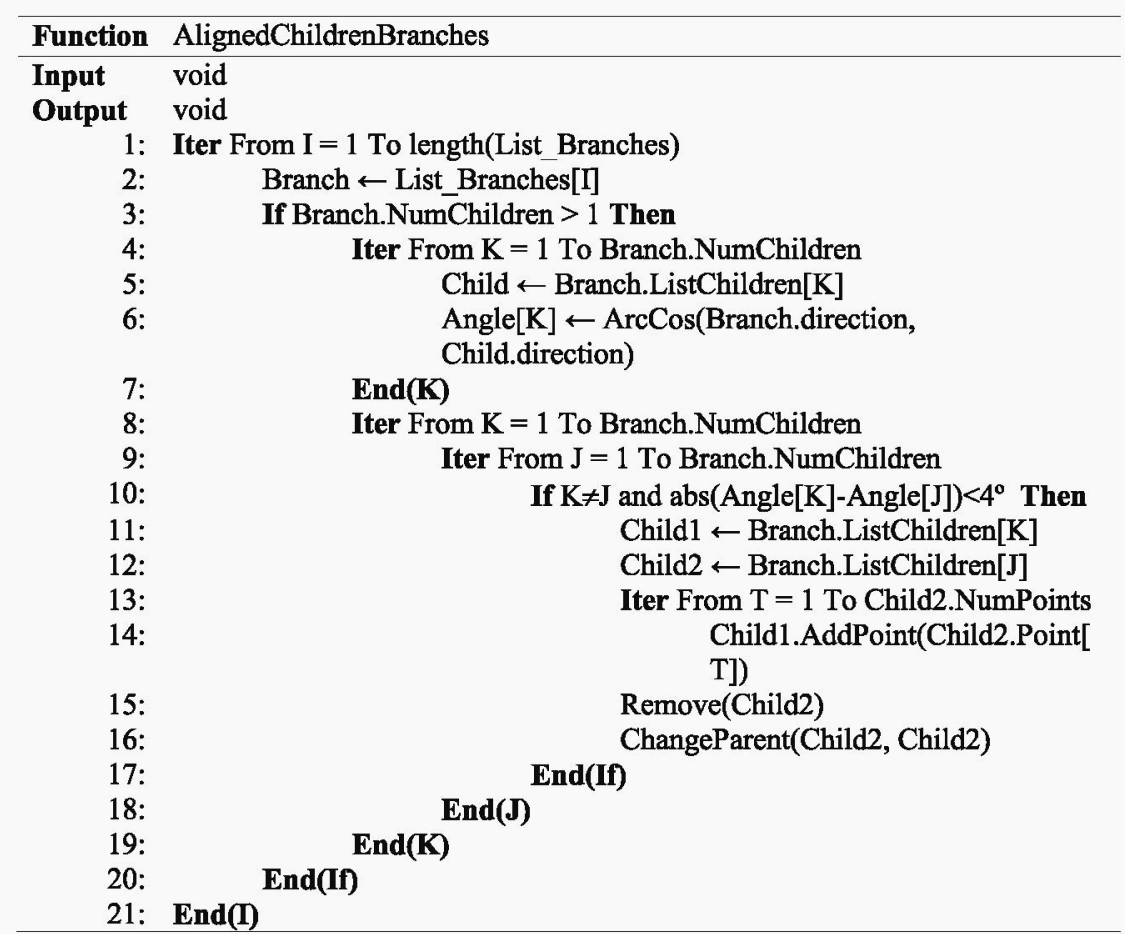


Table 4 - Function that joints a branch with its parent branch when both are aligned.

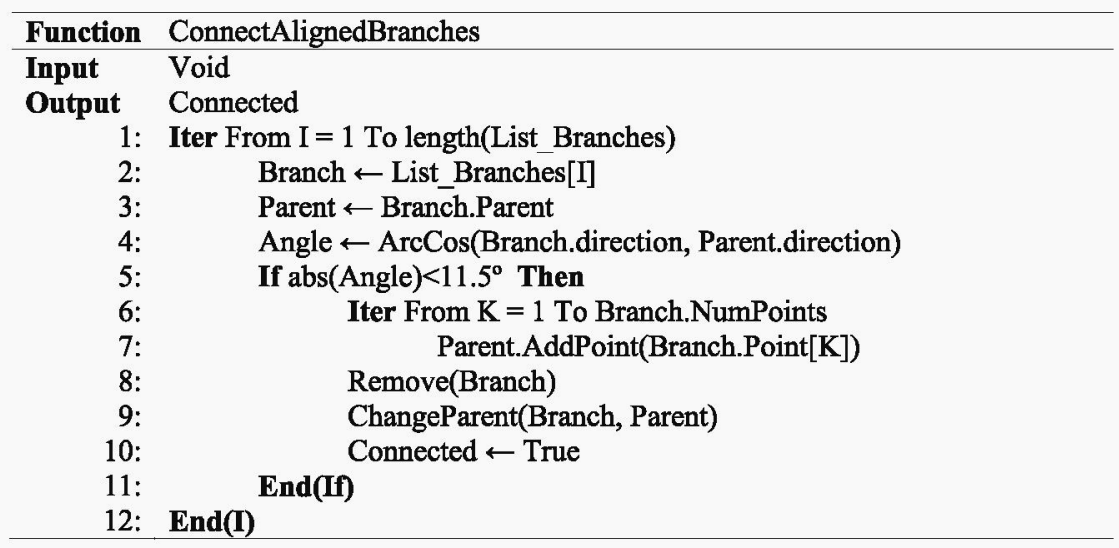

predecessor $(B)$ and successor $(B N)$ branch must be distributed minimising their volume. Galculating the volume of a current branch, knowing $\vec{d}, P_{1}, P_{2}, r$, is a direct operation without additional processing time cost. The clustering process is done by comparing each branch with its successor, which requires less time than comparing each branch with all the rest.

\section{Results and discussion}

Both methods, iterative and least-squared estimate, were compared in a test by generating 100 random directions $\vec{d}$ and, from each direction, an unstructured point cloud. The

Table 5 - Function that balance every branch with its parents to minimize the volume of both.

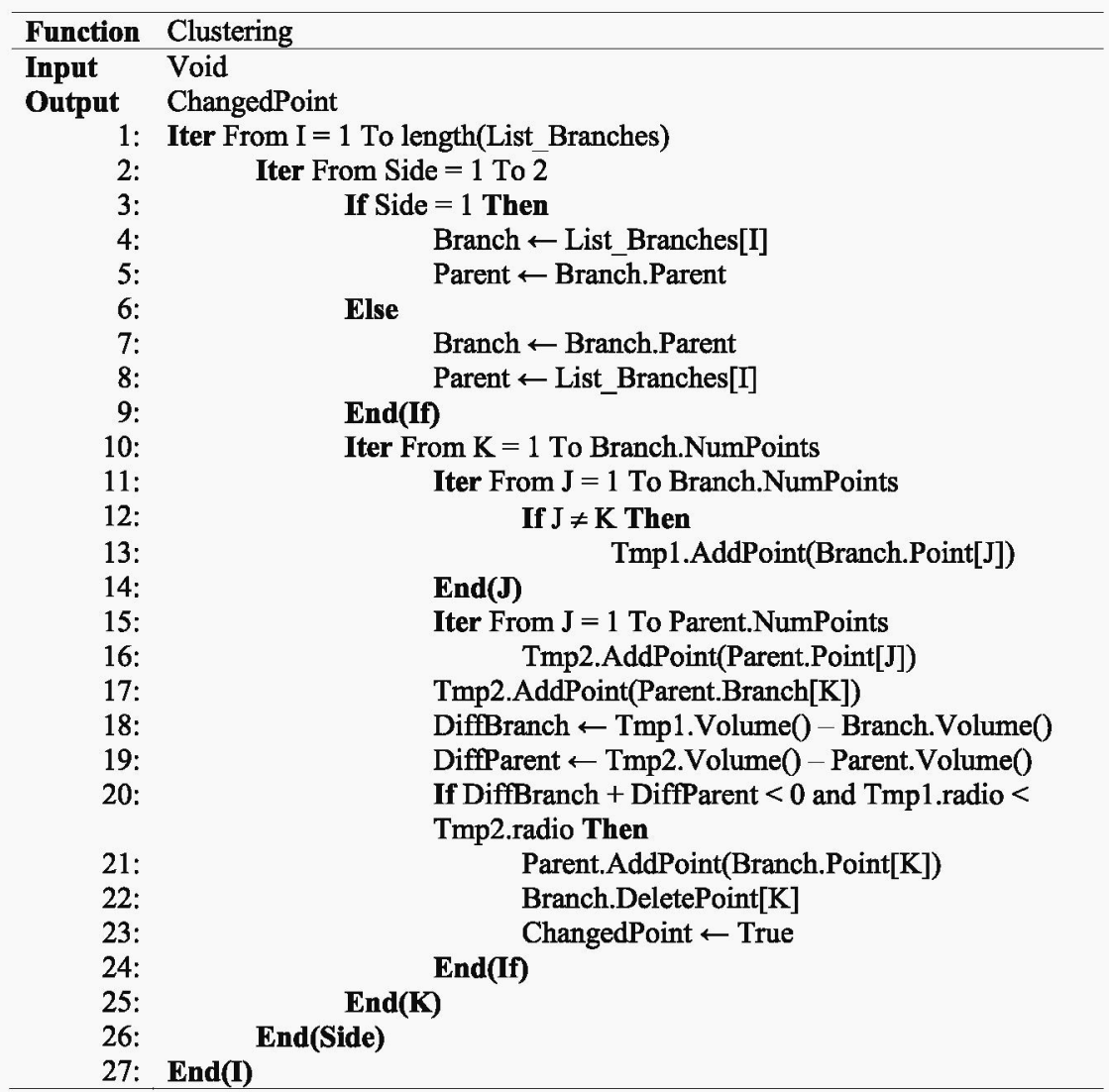


Table 6 - Performance of the Iterative and Lest-squared methods to estimate the cylinders direction.

\begin{tabular}{lccc} 
Method & $\begin{array}{c}\text { Runinng } \\
\text { time (ms) }\end{array}$ & $\begin{array}{c}\text { Average } \\
\text { angle }\left(\vec{d}, \overrightarrow{d^{*}}\right)\end{array}$ & $\begin{array}{c}\text { Standard Deviation } \\
\operatorname{angle}\left(\vec{d}, \overrightarrow{d^{*}}\right)\end{array}$ \\
\hline Iterative & 9.37 & $0.45^{\circ}$ & $0.23^{\circ}$ \\
Least-squared & 0.31 & $0.13^{\circ}$ & $0.06^{\circ}$ \\
& $\vec{d}$ real direction, $\overrightarrow{d^{*}}$ estimated direction \\
\hline
\end{tabular}

results, showing both processing time and accuracy, are shown in Table 6.

The reconstruction of the pear tree model required the lowest values of $k_{r}$ and $n_{s}$ since the generated point cloud was less dense. In the case of the vine, values of $k_{r}$ and $n_{s}$ were smaller than those required for the apple tree since the virtual model had higher ligneous shoot density (Table 7). The number of reconstructed branches and the processing time are shown in Table 8. The reconstruction process, by steps, is shown in Fig. 2.

In the virtual apple tree, the process starts with a sapling which gives rise to the trunk of order 1 and, in the subsequent growth iterations when a branch occurs an order is added to it. The reconstruction process (superposition of branches in the virtual model together with the operation of the MTLS) resulted in over branching of the tree pattern when compared with the original virtual model. Total branch volume was over-estimated, especially in the apple tree reconstruction. As the volume is $h \pi r^{2}$, the error in the radius must be the square root of the error in the volume. In other words, in the initial point cloud, the belonging of a point to a cluster and the cluster hierarchy may have a higher probability than indicated by the initial model. There are also model limitations with respect to the adopted parameters (Table 7). Parameter $t_{d}$ has a stable value, the value of $n_{s}$ is more dependent on the density of scan process. It is required an easy try to verify that the trunk is generated in one cylinder. The algorithm has the advantage that the control of radius with the parent branches is a self-tuning approach.

The lack of accuracy, the reconstructed model is not equal to the SIMLIDAR virtual model, is due to the lack of convergence of the method, defects in the virtual model and the effects derived from the scanning operation. The simulated MTLS operation can generate shadow effects which are aggravated if two branches in the virtual model are superimposed. These shadow effects may cause the reconstruction of a branch to bifurcate to a branch that is, in reality, a continuation of a different branch of the model.

A wrong choice of input parameters can result in an unrealistic reconstruction. Fig. 4 shows three examples where incorrect parameter selection led to a poor reconstruction. If $k_{r}$ is given a large value (Fig. $4 \mathrm{a}$, with $k_{r}=1.22$ and $t_{d}<0.9$ ) branches thinner than normal are obtained, despite the limitations imposed by the constraint that the radius of a branch cannot be greater than its predecessor branch. By taking a value that prevents trunk branching (Fig. $4 \mathrm{~b}$, with $k_{r} \geq 1.23$ and $\left.t_{d}<0.9\right)$, a single unrealistic cylinder is obtained which contains all the points in the point cloud. Choosing a low value of $n_{s}$ (Fig. 4c, withn $n_{s}=4$ ) also results in a poor reconstruction with excessive branching. Based on the De Reffye et al. (1988) branching order, chains of small branches are created resulting in a maximum order in the model much higher than actually exists (in Fig. 4c the maximum order is about 35).

It was estimated that the cost of the algorithm was $O\left(N \cdot \log (N) \cdot \log \left(n_{b}\right)\right)$, where $O$ is the upper limit of growth of the algorithm response time with the increase of $N$, the total number of points in the point cloud, and $n_{b}$ the total number of branches. The main cost of the algorithm is located in the main process (Table 1, lines 4-16), where the iteration was executed $N$ times. Moreover, the FindTheClosestPoint function (Table 2, lines 3-13) function had a cost of $O(\log (N)$. $\left.\log \left(n_{b}\right)\right)$. Nearby points in the kd-tree had a cost of $O(\log (N))$ (Cormen, Leiserson, Rivest, \& Stein, 2009). The estimate

Table 7 - Main parameters used in the analysed reconstructions. $k_{r}$ is the radius factor used to consider if a new point is aligned in a current branch or allows a new branch; $\Delta y$ is the distance between vertical scans; $\Delta \theta$ is the angular resolution of the LiDAR sensor; $t_{d}$ is the parameter of the projection of a point over cylinder axis $\vec{P}_{1} P_{2}\left(t_{d}=0\right.$ when it is projected over $P_{1}$ and 1 if it is projected over $\left.P_{2}\right) ; n_{s}$ is the number of points that freely seed a cylinder when the building of a newbranch starts (this parameter changes depending on the branch order (ord)).

\begin{tabular}{lccccc} 
& \multicolumn{4}{c}{$k_{r}$} \\
\cline { 2 - 5 } & $\Delta y(\mathrm{~cm})$ & $\Delta \theta\left({ }^{\circ}\right)$ & $t_{d}<0.9$ & $t_{d} \geq 0.9$ & $n_{s}$ \\
\hline Apple tree & 1 & 0.5 & 1.05 & 1.10 & $1 ; 3 ; 7 ; 10 ; 9999$ \\
Vine & 1 & 0.5 & 1.05 & 1.10 & $1 ; 3 ; 9999$ \\
Pear tree & 1.5 & 1 & 1.05 & 1.05 & $80 ; 60 ; 40 ; 30 ; 20$ \\
\hline
\end{tabular}

Table 8 - Number of points in the point cloud, number of branches, processing time and volume simulated and rebuilt by the process.

\begin{tabular}{lcccccccc} 
& $\begin{array}{c}\text { Number of } \\
\text { points }\end{array}$ & $\begin{array}{c}\text { Number of } \\
\text { branches }\end{array}$ & $\begin{array}{c}\text { Processing } \\
\text { time (min) }\end{array}$ & $\begin{array}{c}\text { Model } \\
\text { order }\end{array}$ & $\begin{array}{c}\text { Rebuilding } \\
\text { order }\end{array}$ & $\begin{array}{c}\text { Vol. model } \\
\text { (l) }\end{array}$ & $\begin{array}{c}\text { Vol. Rebuilt } \\
\text { (1) }\end{array}$ & $\begin{array}{c}\% \text { Vol. } \\
\text { Error }\end{array}$ \\
\hline Apple tree & 2350 & 164 & 1 & 7 & 11 & 2.80 & 3.63 & $29 \%$ \\
Vine & 4941 & 271 & 2 & 10 & 12 & 6.83 & 7.41 & $8 \%$ \\
Pear tree & 2741 & 278 & 0.5 & & 20 & & & \\
\hline
\end{tabular}




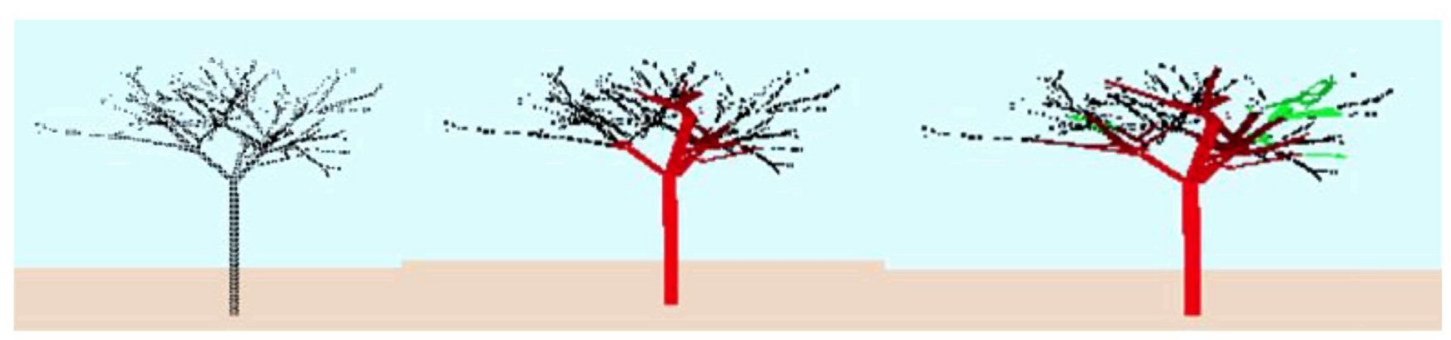

(a)

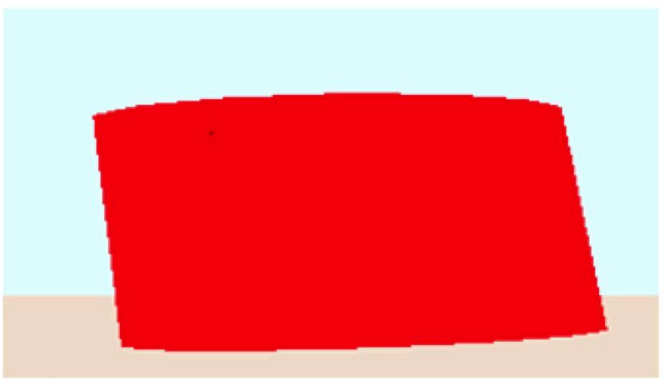

(b)

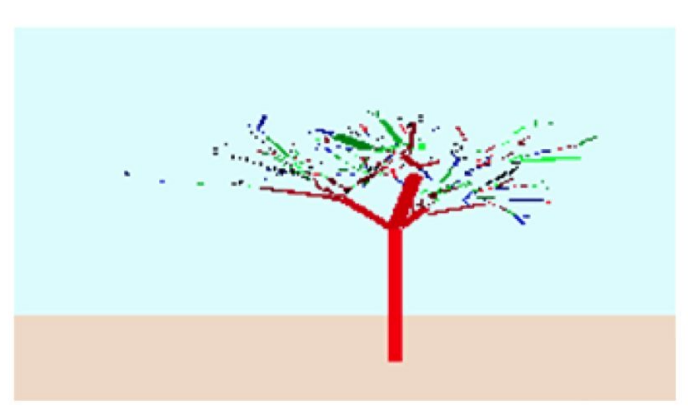

(c)

Fig. 4 - Effect of input parameters on tree model reconstruction: branches of the model wider than those of the measured tree (a); one unrealistic large trunk containing all the points (b); excessive branching (c).

$O\left(\log \left(n_{b}\right)\right)$ was the cost of checking that a point was not closer to the other branches of the model (Table 2, line 7); costing$O\left(n_{b}\right)$ it was underestimated as a result of line 6 . The cost of building a $\mathrm{kd}$-tree (Table 1, line 1) was also $\mathrm{O}(\mathrm{N} \cdot \log (\mathrm{N}))$ (Cormen et al. 2009). The AlignedChildrenBranches procedure, (Table 1, line 17), had a cost of $O\left(n_{b}\right)$, with $n_{b}$ being the total number of branches; the main cost was in the iteration I (Table 3 , line 1) because the cost of the rest of iterations (depending on the number of children of the branch) was small and did not increase with $n_{b}$. In line 18 (Table 1) the ConnectAlignedBranches procedure (Table 1, line 18), had a cost of $O\left(n_{b}\right)$ seen in iteration I (Table 4 , line 1 ); the number of times this function was called was reduced, having an estimated cost of $O\left(n_{b} \cdot \log \left(n_{b}\right)\right)$. Finally, in the Clustering function (Table 5) iteration I (line 1) was performed $n_{b}$ times, while for iteration $\mathrm{K}$ (line 10) the average number of points in a branch was estimated as $N / n_{b}$, resulting in a cost of $O\left(n_{b} \cdot 2 \cdot N /\right.$ $\left.n_{b}\right)=O(N)$ which included, as before, the cost to call the procedure within the main function, $O\left(N \cdot \log \left(n_{b}\right)\right)$. To summarise, by summing all the above results (Table 9, lines 1-6) and considering that the order of magnitude of $n_{b}$ is lower

Table $\mathbf{9}$ - Cost of the functions. Being $\mathbf{N}$ the total number of points of the cloud, $n_{b}$ the total number of branches and $O$ the worst case scenario in terms of computing time according to the dimension of input data.

\begin{tabular}{ll} 
Function & \multicolumn{1}{c}{ Cost } \\
\hline CreateKTree & $O(N \cdot \log (N))$ \\
FindTheClosestPoint & $O\left(\log (N) \cdot \log \left(n_{b}\right)\right)$ \\
AlignedChildrenBranches & $O\left(n_{b}\right)$ \\
ConnectAlignedBranches & $O\left(n_{b} \cdot \log \left(n_{b}\right)\right)$ \\
Clustering & $O\left(N \cdot \log \left(n_{b}\right)\right)$ \\
MainFunction & $O\left(N \cdot \log (N) \cdot \log \left(n_{b}\right)\right)$ \\
\hline
\end{tabular}

than $N$, the proposed algorithm for computational cost is $\mathrm{O}\left(\mathrm{N} \cdot \log (\mathrm{N}) \cdot \log \left(n_{b}\right)\right)$. Thus in the worst case, the computational cost increases in a linear logarithmic order according to the number of points in the cloud.

\section{Conclusions}

Individual tree reconstruction is feasible with a short processing time cost using the proposed algorithm. The disadvantage of the algorithm is the absence of a unique convergence. It is important to correctly adjust the values of the input parameters, in general depending on the MTLS point cloud density. The main parameters are the number of free seed points $\left(n_{s}\right)$ and the radius factor $\left(k_{r}\right)$, which are used to determine whether or not a point is aligned with a branch. The reconstructions obtained correctly matched with the real woody structure of the trees although they are not completely accurate.

The combination of constraints used $\left(n_{s}, k_{r}\right.$ and significant branch radius criterion) avoids divergence of the algorithm and makes the values of the parameters easier to find and less dependent on the type of tree to be reconstructed.

One major advantage of the model is that it only requires a short processing time, and it could therefore be suitable for use in whole orchard reconstruction with several trees trained with common agricultural systems. Orchard reconstruction could be approached by selecting $\mathrm{N}$ tree feet or root points and applying the algorithm to all of them simultaneously. In this case, a kd-tree structure will be required to improve the pointsearching operations. Finally, a clustering process to separate branches that intermingle with each other in different trees would need to be introduced. 


\section{Acknowledgements}

This research was partially funded by the Spanish Ministry of Science and Innovation (SAFESPRAY Project; Grant No. AGL2010-22304-C04-03)

\section{REF EREN C E S}

Auat Cheein, F., \& Guivant, J. (2014). SLAM-based incremental convex hull processing approach for treetop volume estimation. Computers and Electronics in Agriculture, 102, 19-30.

Besl, P., \& McKay, N. (1992). A method for registration of 3D shapes. IEEE Transactions on Pattern Analysis and Machine Intelligence, 14(2), 239-256.

Cormen, T. H., Leiserson, C. E., Rivest, R. L., \& Stein, C. (2009). Introduction to algorithms (3rd ed.) (pp. 248-300). The MIT Press.

Costes, E., Smith, C., Renton, M., Guédon, Y., Prusinkiewicz, P., \& Godin, C. (2008). MAppleT: simulation of apple tree development using mixed stochastic and biomechanical models. Functional Plant Biology, 35, 936-950.

Crouse, M. S., Nowak, R. D., \& Baraniuk, R. G. (1998). Waveletbased signal processing using hidden Markov models. IEEE Transactions on Signal Processing, 46, 886-902.

De Aguiar, E., Stoll, C., Theobalt, C., Ahmed, N., Seidel, H.-P., \& Thrun, S. (2008). Performance capture from sparse multi-view video. ACM Transactions on Graphics, 27(3). Article No. 98.

De Aguiar, E., Theobalt, C., Thrun, S., \& Seidel, H.-P. (2008) Automatic conversion of mesh animations into skeletonbased animations. Computer Graphics Forum, 27(2), 389-397.

De Reffye, P., Edelin, C., Jaeger, M., \& Puech, C. (1988). Plant models faithful to botanical structure and development. Computer Graphics, 22, 151-158.

Delagrange, S., \& Rochon, P. (2011). Reconstruction and analysis of a deciduous sapling using digital photographs or terrestrialLiDAR technology. Annals of Botany, 108, 991-1000.

Durand, J. B., Guédon, Y., Caraglio, Y., \& Costes, E. (2005). Analysis of the plant architecture via tree-structured statistical models: the hidden Markov tree models. New Phytologist, 166, 813-825.

Gorte, B., \& Winterhalder, D. (2004). Reconstruction of laserscanned trees using filter projections in the 3D-raster domain. International Archives of Photogrammetry, Remote Sensing and Spatial Information Sciences, 36(Part 8/W2), 39-44.

Guivant, J., Nebot, E., \& Durrant-Whyte, H. F. (2002). Simultaneous localization and map building using natural features in outdoor environments. Robotics and Autonomous Systems, 20(2-3), 79-90.

Henning, J., \& Radtke, P. (2006). Detailed stem measurements of standing trees from ground-based scanning LIDAR. Forest Science, 52(1), 67-80.

Iagnemma, K., Kang, S., Shibly, H., \& Dubowsky, S. (2004). Online terrain parameter estimation for wheeled mobile robots with application to planetary rovers. Robotics, IEEE Transactions, 20(5), 921-927.

Lloyd, S. (1982). Least square quantization in PCM. IEEE Transactions on Information Theory, 28, 129-137.

Lukács, G., Martin, R., \& Marshall, D. (1998). Faithful least-squares fitting of spheres, cylinders, cones and tori for reliable segmentation. In ECCV '98: Proceedings of the 5th European Conference on Computer Vision-Volume I (pp. 671-686). SpringerVerlag.

Marshall, A. D., Lukács, G., \& Martin, R. (2001). Robust segmentation of primitives from range data in the presence of geometric degeneracy. IEEE Transactions on Pattern Analysis and Machine Intelligence, 23(3), 304-314.
Méndez, V., Catalán, H., Rosell, J. R., Arnó, J., \& Sanz, R. (2013) LiDAR simulation in modelled orchards to optimise the use of terrestrial laser scanners and derived vegetative measures. Biosystems Engineering, 115, 7-19.

Méndez, V., Catalán, H., Rosell, J. R., Arnó, J., Sanz, R., \& Tarquis, A. (2012). SIMLIDAR - simulation of LiDAR performance in artificially simulated orchards. Biosystems Engineering, 111(1), 72-82.

Mizoue, N., \& Masutani, T. (2003). Image analysis measure of crown condition, foliage biomass and stem growth relationships of Chamaecyparis obtusa. Forest Ecology and Management, 172, 79-88.

Nebot, E., \& Durrant-Whyte, H. (1999). Initial calibration and alignment of low cost inertial navigation units for land vehicle applications. Journal of Robotics Systems, 16(2), 81-92.

Neira, I., Tardos, I. D., \& Castellanos, J. A. (2003). Linear time vehicle relocation in SLAM. Proceedings. ICRA '03. In IEEE International Conference on Volume 1 (pp. 427-433). Robotics and Automation, 2003.

Pfeifer, N., Gorte, B., \& Winterhalder, D. (2004). Automatic reconstruction of single trees from terrestrial laser scanner data. In Proceedings of 20th ISPRS Congress: Geo-imagery Bridging Continents, 12-23 July, Istanbul, Turkey (pp. 114-119).

Phattaralerphong, J., \& Sinoquet, H. (2005). A method for 3D reconstruction of tree crown volume from photographs: assessment with 3D-digitized plants. Tree Physiology, 25, 1229-1242.

Phattaralerphong, J., \& Sinoquet, H. (2007). Tree analyser: software to compute tree structure parameters from photographs. User manual. PIAF-INRA http://www2.clermont.inra.fr/piaf/eng/ download/download.php.

Preuksakarn, G., Boudon, F., Ferraro, P., Durand, J. B., Nikinmaa, E., \& Godin, C. (2010). Reconstructing plant architecture from 3D laser scanner data, 6th International Workshop on Functional-Structural Plant Models, Davis: USA.

Rabbani, T., \& Heuvel, F. (2005). Efficient Hough transform for automatic detection of cylinders in point clouds. ISPRS WG III/3, III/ 4, V/3 Workshop "Laser scanning 2005", Enschede, the Netherlands, September 12-14.

Reulke, R., \& Haala, N. (2005). Tree species Recognition with Fuzzy texture parameters. Combinatorial image analysis, Springer. Lecture Notes in Computer Science, 3322, 607-620.

Rosell, J. R., Llorens, J., Sanz, R., Arnó, J., Rỉbes-Dasi, M., Masip, J., et al. (2009). Obtaining the three-dimensional structure of tree orchards from remote 2D terrestrial LIDAR scanning. Agricultural and Forest Meteorology, 149, 1505-1515.

Sanz-Cortiella, R., Llorens-Calveras, J., Escolà, A., Arnó-Satorra, J., Ribes-Dasi, M., Masip-Vilalta, J., et al. (2011). Innovative LIDAR 3D dynamic measurement system to estimate fruit-tree leaf area. Sensors, 11, 5769-5791.

Shlyakhter, I., Rozenoer, M., Dorsey, J., \& Teller, S. (2001). Reconstructing 3D tree models from instrumented photographs. IEEE Computer Graphics and Applications, 21, 53-61.

Simonse, M., Aschoff, T., Spiecker, H., \& Thies, M. (2003). Automatic determination of forest inventory parameters using terrestrial laser scanning. In Proceedings of ScandLaser Workshop, 3-4 September 2003, Umea, Sweden (pp. 251-257).

Tan, P., Fang, T., Xiao, J., Zhao, P., \& Quan, L. (2008). Single image tree modeling. ACM Transactions on Graphics, 27. http:// dx.doi.org/10.1145/1409060.1409061. Article 108.

Verroust, A., \& Lazarus, F. (2000). Extracting skeletal curves from 3D scattered data. The Visual Computer, 16(1), 15-25. February 2000 .

Yan, D.-M., Wintz, I., Mourrain, B., Wang, W., Boudon, F., \& Godin, C. (2009). Efficient and robust tree model reconstruction from laser scanned data points. CAD/Graphics, 2009, 572-575. 